\title{
Underwater Image and Signal Processing
}

\author{
Neha Chavan \\ Electronics and Telecommunication Enginnering \\ P.C.C.O.E.R, Ravet \\ Pune, India \\ Sanket Darur \\ Electronics and Telecommunication Enginnering \\ P.C.C.O.E.R, Ravet \\ Pune, India
}

\begin{abstract}
This paper proposes study of underwater image processing as well as signal processing. It is widely used in underwater applications like, underwater object detection, submarine communication, to observe the sea floor which is included in autonomous underwater vehicles (AUVs), unmanned underwater vehicles (UUVs), and in situ ocean sensor networks. This project is concerned with various techniques of underwater image and signal processing. We present an overview of various underwater image-processing approaches, such as underwater image de-scattering underwater image color restoration, and underwater image quality assessments along with signal processing which covers, acoustic signal transmission, reception, filtering and representation. In this paper, objective is to acquire a given audio signal to communicate through underwater to give the acquired signal in time and frequency representation. We, hence, try to provide with underwater image and signal processing which can be useful and low- cost solution.
\end{abstract}

Keywords-Hydrophone, Image, Signal, Underwater Processing.

\section{INTRODUCTION}

There are various projects like underwater bridge construction in the world which requires survey of underwater area. Numerous systems like surveillance require aid of underwater communication. In order to make these tasks easy for an user who has little or no knowledge of underwater image and underwater signal processing we introduce a cost effective system made of combining underwater image capturing, underwater image processing, underwater acoustic signal transmission, underwater acoustic signal processing and of an easy to use interface like, GUI. This can simplify the tedious tasks of underwater study and underwater communication. Thus, in acoustic processing, we transmit audio signal and then when the signal is received, it is filtered and then represented as frequency response, magnitude response, etc. For this communication hydrophone is used. Hydrophone is a simple device made of piezoelectric crystal which works as a transmitter and as receiver too. While in image processing we go for image capturing, image restoration, image enhancement and colorcorrection.

\author{
Chinmayee Chitnis \\ Electronics and Telecommunication Enginnering \\ P.C.C.O.E.R, Ravet \\ Pune, India \\ Rupali Kawade \\ Electronics and Telecommunication Enginnering \\ P.C.C.O.E.R, Ravet \\ Pune, India
}

\section{METHODOLOGY}

In this paper we have discussed the methods to achieve the Underwater Image and signal processing. For Image processing there are many methods used in practice, out of which image processing using MATLAB is used on a large scale. For underwater Images we use an Endoscopic Camera. Due to this there is no need for external light source with the camera. Once the images are captured they are sent through a GUI for Image processing. The GUI uses an algorithm which contains different MATLAB functions for image processing. After processing of Images through GUI we get a series of enhanced images from which we can choose the required result.

Signal processing on the other hand, is useful for underwater sound detection and various signals that are present underwater. The signal processing takes place using a HYDROPHONE. The Hydrophone consists of a piezoelectric element which receives vibrations underwater and converts them into electric signals. These electrical signals will give information about the signals present underwater. We can either use a single Hydrophone or an array of Hydrophone for receiver. The received signals are processed on MATLAB. We can perform filtering, and try to recover the original signals. We can also take a spectrogram of the signal and study the frequencies present in the signal at different levels.

\section{A. BLOCK DIAGRAM}

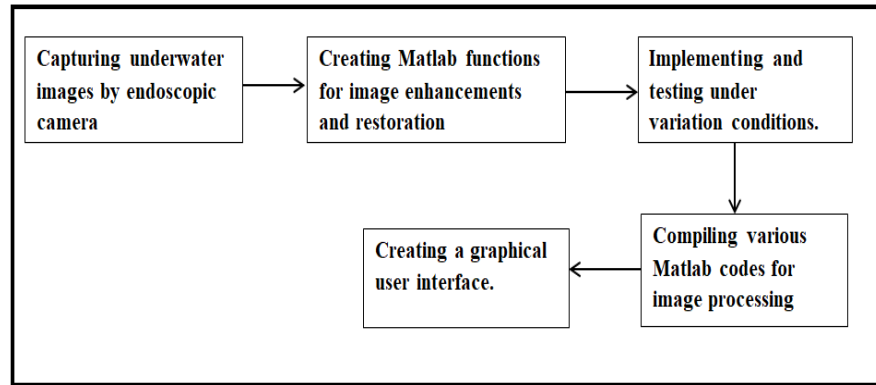

Fig.1

Capturing underwater image by endoscopic camera: An endoscopic camera is basically a little camera along with a long cable which can be used to view tiny areas on a big screen.

Creating MATLAB functions for image enhancement and restoration: Image enhancement uses qualitative 
subjective criteria to produce a more visually pleasing image and they do not rely on any physical model for the image formation. We have used some methods like, white balance, enhance contrast, object detection, luminance adjustment, chromaticity adjustment, saliency detection, etc.

Implementation and testing under various conditions: Various codes and functions are tested for Image enhancement. This will help us restore the color balance, white balance, image filtration and balance the RGB components.

Compiling various MATLAB codes for image processing: Creating different codes on MATLAB based on the functions created for image enchantment. These codes are compiled based on the various functions tested earlier under different conditions. We need to decide which function will be suitable for the image captured.

Graphical user interface: A GUI is made for carrying out these various functions by adding buttons for each of these functions. Also, using a GUI we can easily observe which image processing function or code should be used for image enhancement or extraction of the original image through the captured underwater image.

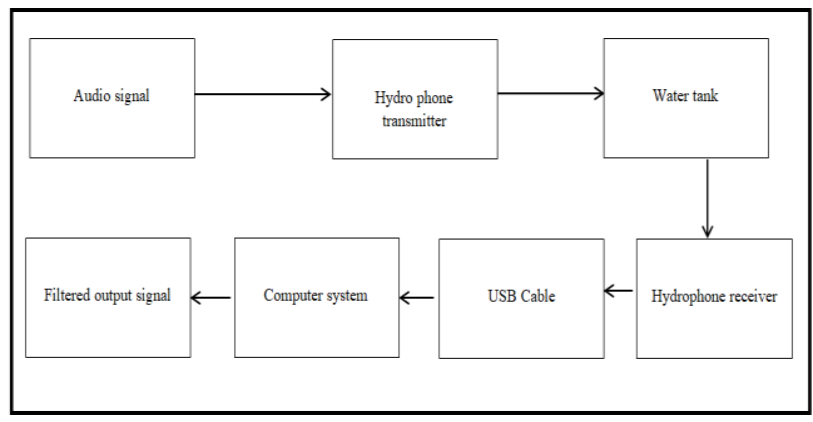

Fig.2

Audio signal: Audio signals are electronic representations of sound waves-longitudinal waves which travel through air, consisting of compressions and rarefactions. The energy contained in audio signals is typically measured in decibels. The audio signal which is of $20-20 \mathrm{kHz}$ frequency is transmitted to the hydrophone transmitter.

Hydrophone Transmitter: The audio signal is transmitted to the hydrophone transmitter basically hydrophone is used to convert a electrical signal into sound waves

Water tank: In our project water tank is represented as a water body. The hydrophone is placed under water for signal transmission and reception. Due to water, noise is added to audio signal.

Hydrophone Receiver: The audio signal is received to the hydrophone transmitter Basically hydrophone is used to convert a sound wave into electrical signal by detecting changes in pressure in the surrounding environment.

USB Cable: It provides a physical connection between the receiver and computer system.

Computer system: It is used for signal processing. It stores the received signals and performs various functions regarding signal processing such as filtering, spectrogram, etc.

\section{B. Sotware Design}

We compiled and implemented different functions on MATLB software. These functions are combined into a GUI. Parameters to these functions can be varied with the help of GUI at a user's will. Any user can produce the outputs according to his/her requirement with help of various processing functions available in GUI. Following are some tasks a user can perform using software part of this system.

\section{Hardware Design}

In this system the most important hardware used is hydrophone transmitter and receiver. A hydrophone is a microphone designed, here, to be used underwater for recording or listening to underwater sound. Most hydrophones are based on a piezoelectric transducer that generates an electric potential when subjected to a pressure change, such as a sound wave. When an electrical voltage is applied to the crystalline ceramic material, the crystalline structure aligns, becomes anisotropic, and carries an electrical charge. Use of a piezoelectric transducer as an alternative to crystal material is now a popular option in modern-day hydrophone technology. A hydrophone with a single transducer has a circular conical shape that reflects incoming sound waves which in turn allows the hydrophone to be positioned at varying locations and depth. The only problem with a single transducer is that signals from other directions interfering with the main signal cannot be subtracted and this can obscure data about the location of the desired signal. Array hydrophones (streamers - built of multiple transducers) are all wired to receive a large sound signal collectively. The transducers are packed together in a tube with oil, which aids the collection of pressure waves entering the hydrophone. Pre-amplifiers are often used to enhance the electrical signal and limit the potential of noise contamination from additional components to the hydrophone.

\section{LITREATURE SURVEY}

Underwater image processing and data acquisition covers two wide areas of image processing and signal processing. It is widely used in underwater applications like, underwater object detection, submarine communication, to observe the sea floor which is included in autonomous underwater vehicles (AUVs), unmanned underwater vehicles (UUVs), and in situ ocean sensor networks. This project is concerned with various techniques of underwater image and signal processing. India is developing country. Following are few major underwater projects proposed, in India: India could soon have its first underwater train in Kolkata. The Navy has launched the Scorpene class submarine Vela, the fourth of six underwater warships being built in India with French collaboration. India has undertaken many more underwater ventures like this.

[5]Various researches show that due to absorption and scattering of underwater the images obtained suffers an 
information loss. The study of underwater is very important to find the many algorithms have been derived to enhance an underwater image but no method gives a clear vision of human eye. Many methods found suffers a disadvantage that the clarity reduces as the depth of the water increases this is due to the fading of colors in underwater. To solve this problem, they found a solution having a non-reference underwater image enhancement method the UICCS method. The three main attributes of an image are its color, contrast and sharpness. In this method we modify the attributes of the image step by step to attain a clear image.

[6] Following are few constraints in underwater imaging. First, due to the medium, scattering always causes a blurring effect in underwater photography; this rarely occurs in land photography. Second, wave- length absorption usually causes a color reduction in the captured images, which rarely occurs in air. Third, except for electronic noise, the sediments in the water also affect high dimensional imaging.

[7] The underwater scenes are captured based on exposure bracketing, which is a technique used to acquire multiple images with different exposure times. The long-exposure image is useful for sufficiently acquiring red spectrum information that is particularly attenuated in underwater scenes. In contrast, pixel values in the green and blue channels in the long-exposure image will saturate be- cause the green and blue components are unlikely to attenuate compared to the red one. To avoid this, the images are taken such that the green and blue pixel values are from the short-exposure image. In this way, the images are fused such that they contain adequate spectral information of underwater scenes.

[8] According to the characteristic of underwater optical imaging, the blue light travels the longest in the water because of its shortest wavelength, followed by the green light and then the red light. Finally, among the aboveselected brightest pixels, the pixel with the maximum bluered difference in the input image is regarded as the estimated global background light for guaranteeing the robustness of that proposed algorithm.

[9] However, acoustic signal are affected by ambient noise (consists of man made noise and natural noise) during transmission and hence, identification and recognition of required signal is equally important. Here, ambient noise refers to the sound received by the Omni-directional sensor which is not from the sensor itself or from the manner in which it is mounted. Following are few types of noise.

- Hydrodynamic Noise: It occurs due to movement of water because of tides, winds, currents, storms. Level of this noise depends on the surface of the sea.

- $\quad$ Seismic Noise: It is caused due to movements of land under or near the sea.

- $\quad$ Ocean traffic: It is generally sound caused due to shipping. It depends on number of ships in the area having receiver and distance of ships from the receiver. As, distance increases, only low frequencies propagate (approx. $200 \mathrm{~Hz}$ ) and high frequencies are attenuated.

- Biological Noise: It constitutes of animals, distance shipping, rain, wind, bubbles, etc.
[10] Acoustic sensor data from a single hydrophone onboard a Slocum glider were recorded during the glider's deployment in a mock undersea surveillance mission. This shallow water environment, which has a typical depth of $90 \mathrm{~m}$, was characterized by good acoustic propagation conditions and low ambient sea noise levels. Post processing of the data resulted in the detection of a helicopter, a surface vessel and the presence of tone burst transmissions from an underwater sound beacon. Underwater Sound Beacon Transmissions The output of the single hydrophone onboard the glider is sampled every $10 \mu \mathrm{s}$, that is, at a sampling rate of $100 \mathrm{kHz}$. For the detection of tone burst transmissions from an underwater sound beacon, the data are down sampled to $15 \mathrm{kHz}$. The hydrophone onboard the undersea glider also sensed the presence of strong underwater acoustic transient signals, which were identified as being of biological origin These transient signals are echolocation sound pulses (or clicks) emitted by Indo-Pacific bottlenose dolphins.

[11] A user friendly UI (user interface) is written to enhance the utilization. A full year of data are analyzed with the detector, and the results are satisfactory in the robustness the detection rate and the efficiency of analysis of large amount of data.

-Hydrophone's Quality:

The quantity of acoustic data in each day is about $130 \mathrm{~GB}$, wave format, and separated to zero and $20 \mathrm{~dB}$, two kinds of the gain's setting. By analyzing the variation of minutes and hours, we found some problems in raw data. The recording system is usually made a single wave file about 30 seconds. We found that some files will be less than 30 seconds (CASE1). Sometimes the system needs to restart due to the instability. In order to fix some broken files which is less than 30 seconds, the system will fill zero in the lacking time. Other situation, it will skip to make files even for tens of minutes. (CASE2) However, the system has been operated more smoothly and stably during 2012. If CASE1's problem happened or the zero in the file is more than 5 seconds, the detector will pass that file. For the reason, we set the limit resolution is 30 seconds.

[12] In Sonar systems, for underwater target detection cylindrical arrays are used. For reducing calculation, each line of hydrophones at the same azimuth direction aligned with the cylinder axis are hardwired together in practice and thus, each line array with omnidirectional sensors becomes a directional sensor steered to broadside. As a result, the cylindrical array can be treated as a circular array with directional elements. The cylindrical array is made up of several circular hydrophone arrays. For reducing the computational load, the hydrophones in each line are wired together and thus the cylindrical array is converted to a circular array with directional elements. The acoustic signals by underwater vessels are almost at zero elevation. Thus, without suppressing the signal we can reduce the ambient noise. Because the hydrophones in each line are hardwired together, the incident angle of the signal is assumed to be horizontal, which means the elevation angle is $0 \theta=0^{\circ}$. Then the cylindrical array can be viewed as that of a circular array. 
[13] Hydrophone is a type of microphone that is used in underwater for recording and also for listening to the underwater sound signals. Generally, Hydrophones are based upon the transducer named Piezo electric element that can convert the vibrational effects into an electrical signals. Underwater communications are taking place at a frequency of $10 \mathrm{~Hz}-1 \mathrm{MHz}$. The field of underwater acoustic communication grows rapidly because underwater acoustic channel is used in many applications like Seismic monitoring, Pollution monitoring.

[14] The received signal, which is emitted from an underwater source, has a multipath time delay caused by the surface/bottom reflection. Multipath propagation is a drawback of a positioning system using the time difference of arrival for estimating the target bearing and range. For source position estimation in a multipath environment, other systems require the advantages of various signal processing systems. The signals transmitted from an underwater object undergo different propagation paths that cause distortion of the signals through reflections and diffractions on the sea surface and bottom.

\section{RESULTS}

Image processing is the technique to convert an image into digital format and perform operations on it to get an enhanced image or extract some useful information from it.

Following are the results for image processing performed on a underwater image
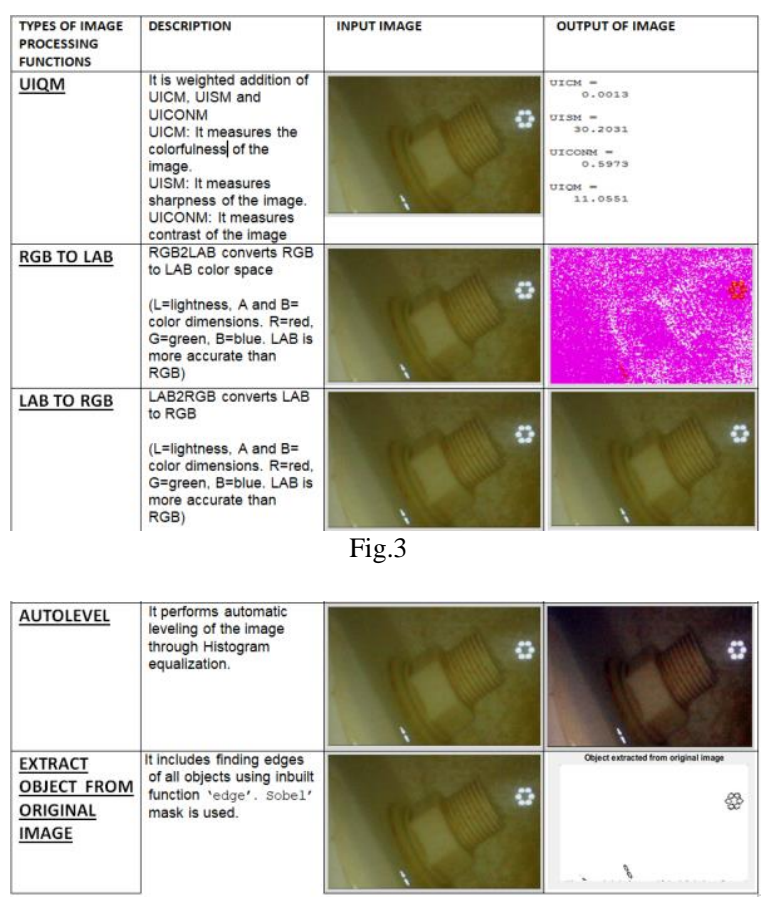

Fig. 4

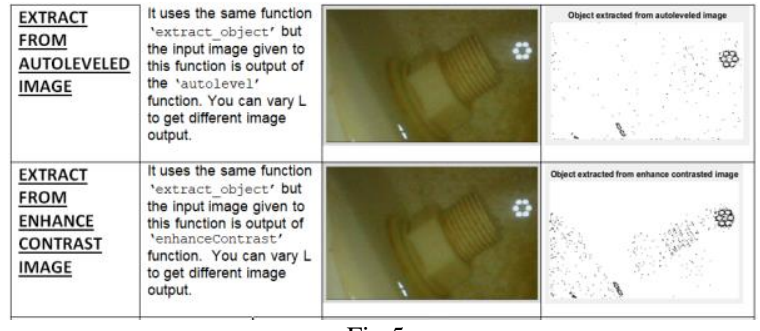

Fig.5

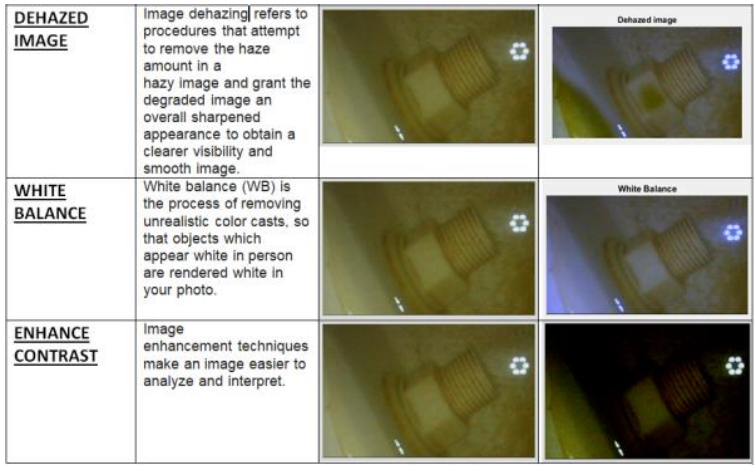

Fig.6

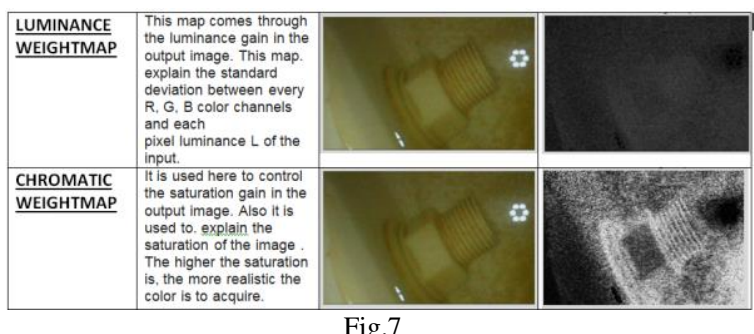

Fig.7

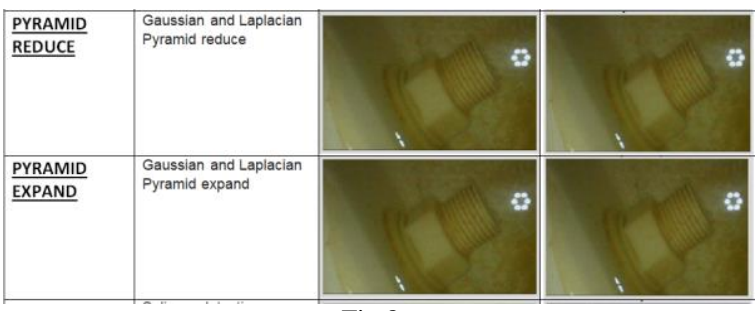

Fig.8

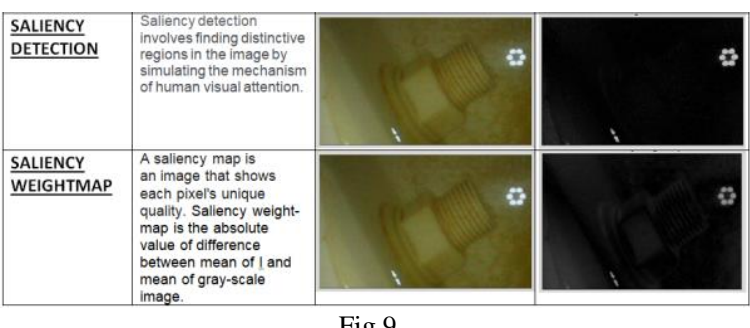

Fig.9

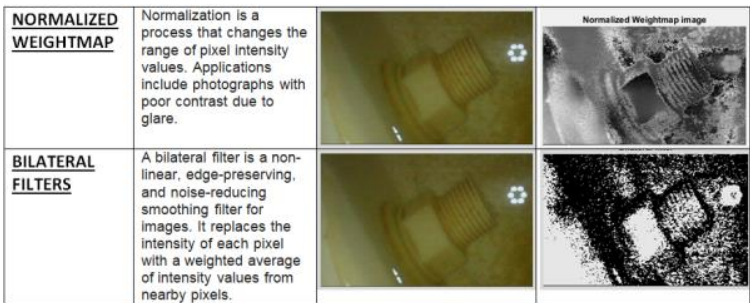

Fig.10 
Various functions were built for audio signal processing. With help of these audio functions we can filter the audio signals, remove and add noise, and calculate frequency domain as well as time domain.

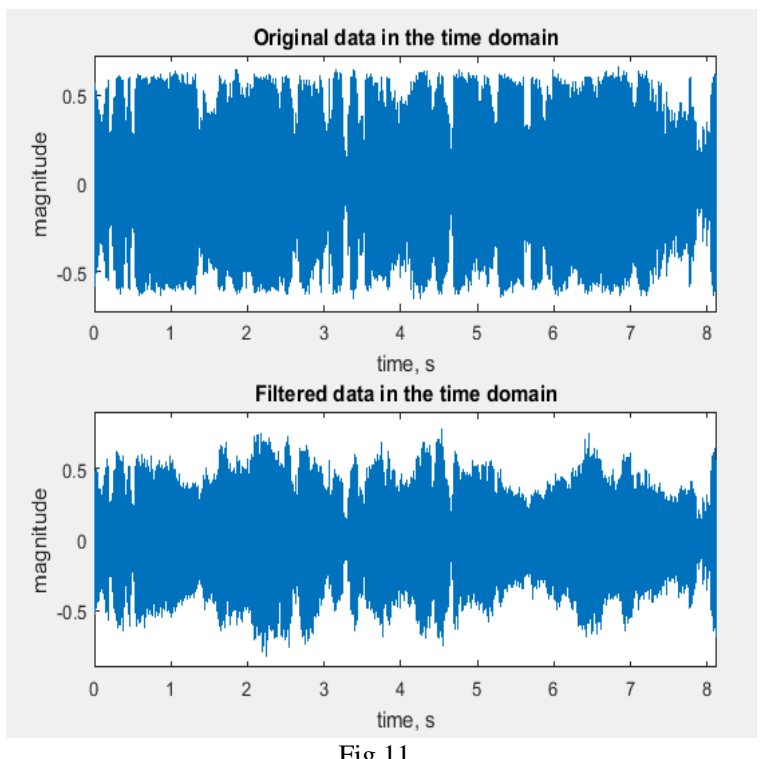

Fig.11
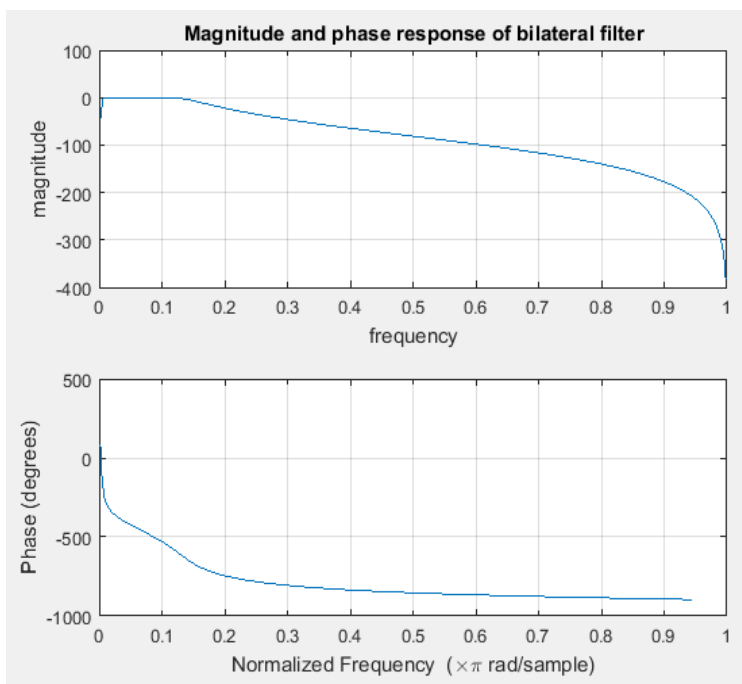

Fig. 12

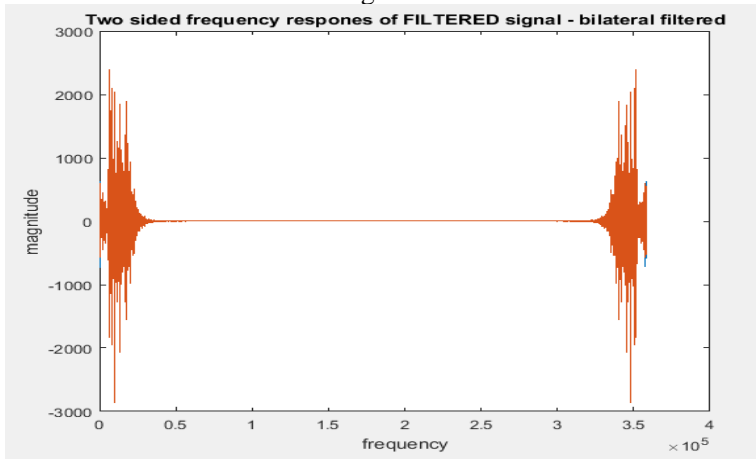

Fig. 13

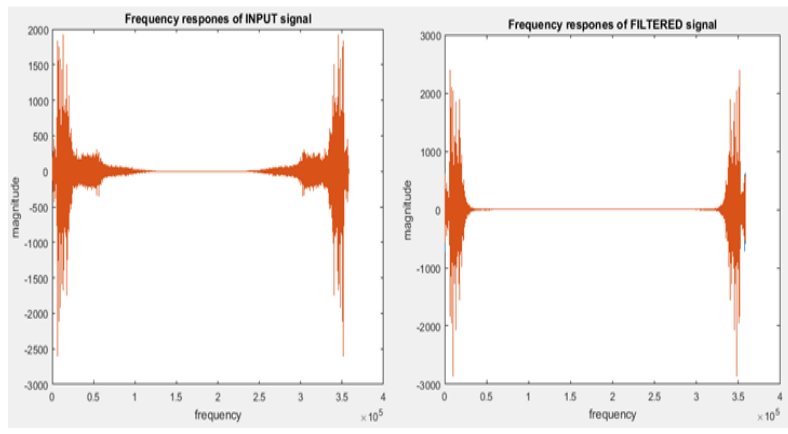

Fig.14

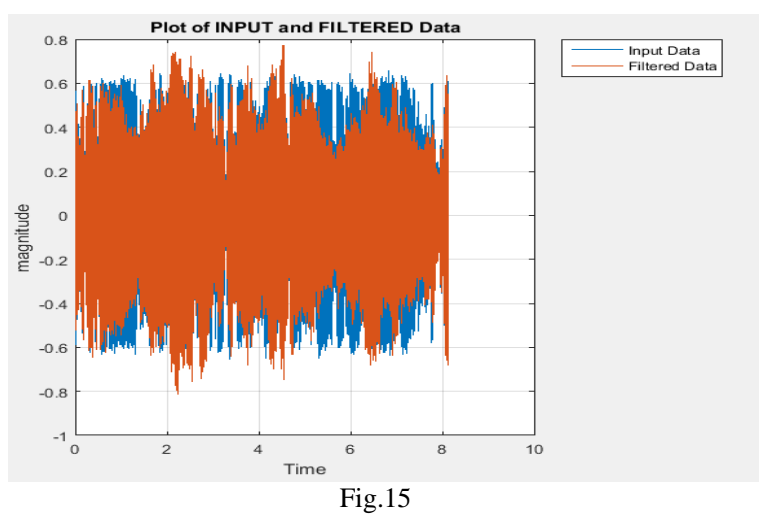

CONCLUSION

In this system, we are building a complete system of hardware and software to transmit and receive audio signal in water. Hardware consists mainly of hydrophone as transmitter and receiver while software consists mainly of MATLAB for processing of received signal. Images are captured by endoscopic camera and given to the computer system where it is processed by various MATLAB functions. Finally, we get a complete system that can perform underwater communication and can process underwater signal and image.

\section{ACKNOWLEDGMENT}

This paper and the research behind it would not have been possible without the exceptional support of our college Pimpri Chinchwad College of Engineering \& Research, Ravet, for providing research environment and also for their kind inspiration. We are also grateful for the insightful comments offered by the anonymous peer reviewers.

\section{REFERENCES}

[1] Article in economic times, "India's first underwater train to be launched in Kolkata soon" link: https://economictimes.indiatimes.com/industry/transportation/r ailways/indiasfirst-underwater-train-to-be-launched-inkolkatasoon/articles how/7060

[2] Article in economic times, "INS Vela: Indian Navy launches

[3] fourth Scorpene-class submarine Vela in Mumbai" link: https://economictimes.indiatimes.com/news/defence/insvelaindian-navy-launches-fourth-scorpene-class-submarinevela-inmumbai/videoshow/69203110.cms

[4] Article in Bharat Shakti, "Adani Group Puts in Last-Minute 
[5] Bid for Indian Navy's Rs 45,000-Crore Submarine Project" link:

[6] https://bharatshakti.in/adani-group-puts-in-last-minute-bid-forindianavys-rs-45000-crore-submarine-project/

[7] Article in The Print, "India to launch Rs 200 crore deep sea research project to help with underwater mining" link: https://theprint.in/india/india-to-launch-rs-200-crore-deep-searesearchproject-to-help-with-underwater-mining/285182/

[8] Joel Fathimson. J, Bibis.S, Aswanth.R, Gayatri.S. , Underwater Image

[9] Restoration Using UICCS Method in MATLAB , International Journal of New Technology and Research (IJNTR), 2018

[10] Huimin Lu, Yujie Li, Yudong Zhang, Min Chen, Seiich Serikawa, Hyoungseop Kim, Underwater Optical Image Processing, USA, 2017

[11] Kohei Nomura, Daisuke Sugimura And Takayuki Hamamoto, Color Correction Of Underwater Images Based On MultiIluminant Estimation With Exposure Bracketing Imaging, Tokyo, 2017

[12] Chongyi Li, Jichang Guo, Shanji Chen, Yibin Tang, Yanwei Pang, JianWang, Underwater Image Restoration Based On Minimum Information Loss Principle And Optical Properties Of Underwater Imaging, IEEE International Conference On Image Processing, 2016
[13] Andrew C. Singer, Jill K. Nelson, Suleyman S. Kozat, Signal Processing for Underwater Acoustic Communications, IEEE Communications Magazine, 2009

[14] Brian G. Ferguson, Kam W. Lo and Joshua D. Rodgers, Sensing the Underwater Acoustic Environment with a Single Hydrophone Onboard an Undersea Glider, Australia, 2010

[15] Yin-Ying Fang, Meng-Chu Liu, Shih-En Chou, Chifang Chen Chien-Kang Huang, Event Detection of Underwater Acoustic Data from MACHO hydrophone, Department of Engineering Science and Ocean Engineering, National Taiwan University, 2017

[16] Shaohao Zhu, Yixin Yang, Yong Wang, Qiulong Yang, Xidang Yan, Performance of a Cylindrical Hydrophone Array for Practical Use, China, 2017

[17] TS Vishnu Priya, G.Vinitha Sanchez, N.R.Raajan, Underwater Acoustic Communication Using MIMO Hydrophone, India, 2018

[18] Min Seop Sim, Byoung-Nam Kim, Eung Kim, and BokKyoung Choi, Experimental and Simulation Analysis of Underwater Acoustic Source Localization using Closely Spaced Hydrophone Pairs, Korea Institute of Ocean Science \& Technology (KIOST) Ansan-si, Republic of Korea, 2017 